\title{
Kernos
}

Revue internationale et pluridisciplinaire de religion grecque antique

15 | 2002

Varia

\section{Silvia LANZI, Theos Anaitios. Storia della teodicea da Omero ad Agostino}

\section{André Motte}

\section{OpenEdition \\ Journals}

\section{Édition électronique}

URL : http://journals.openedition.org/kernos/1452

DOI : 10.4000/kernos. 1452

ISSN : 2034-7871

\section{Éditeur}

Centre international d'étude de la religion grecque antique

Édition imprimée

Date de publication : 1 janvier 2002

ISSN : 0776-3824

\section{Référence électronique}

André Motte, «Silvia LANzı, Theos Anaitios. Storia della teodicea da Omero ad Agostino », Kernos [En ligne], 15 | 2002, mis en ligne le 22 avril 2011, consulté le 22 septembre 2020. URL : http:// journals.openedition.org/kernos/1452 ; DOI : https://doi.org/10.4000/kernos.1452 
traitant des sacrifices de chiens est particulièrement intéressant pour l'étude des rituels lucaniens relatifs aux "dépôts d'offrandes" scellés par des ossements canins brûlés. Le parallélisme avec l'un des botbroi de l'Héraion du Sele ouvre par ailleurs des perspectives de recherches intéressantes sur les actes rituels et leurs circonstances ainsi que sur le choix des animaux sacrifiés en milieu lucanien. On déplorera toutefois la médiocrité de la qualité des clichés couleur et noir et blanc (impression complètement floue), le manque de clarté des cartes et l'absence de certains plans. Ainsi, la carte de la Lucanie présentée au chapitre I est non seulement trouble mais la taille du caractère typographique choisi pour les noms des villes rend toute lecture impossible. Il en est de même pour les noms et la légende de la carte de la figure 5 au chapitre II relative à la distribution des nécropoles archaïques de la région de Torre di Satriano. On aurait également aimé retrouver un plan général du site (éventuellement repris de l'ouvrage de R.R. Holloway, ill. 2 et complété par les données récentes) qui aurait aidé le lecteur à localiser plus facilement le sanctuaire par rapport au secteur d'habitat et des nécropoles (les seuls plans du lieu de culte étant ceux des secteurs de fouilles présentés en dehors de tout contexte topographique). Espérons donc que le rapport de fouille définitif s'accompagne de nombreuses cartes utiles et lisibles ainsi que de photographies de qualité. Une autre remarque porte sur le manque de soin apporté aux références et à la bibliographie. À plusieurs reprises des références citées dans un texte n'ont pas été reprises dans la bibliographie générale en fin de volume (par ex. Lejeune 1967, 1971 et 1972; Lavagnini 1923; Lepore 1972 ou encore Prosdocimi 1988). En dehors de ces quelques reproches, Rituali per una dea lucana reste un ouvrage important et indispensable pour qui s'intéresse aux cultes lucaniens, que ce soit pour la topographie des sanctuaires, l'architecture des édifices sacrés ou encore les activités rituelles, qu'il s'agisse des banquets ou de l'ensevelissement des offrandes votives.

Sandrine Ducaté-Paarmann (Université de Paris IV-Sorbonne)

Silvia Lanzi, Theos Anaitios. Storia della teodicea da Omero ad Agostino, présentation de G. Casadio, Roma, Il Calamo, 2000. 1 vol. $16,5 \times 24 \mathrm{~cm}, 349 \mathrm{p}$. (Bibliotheca di Storia delle religioni). ISBN : 88-86148-68-2.

Theos anaitios, «Dieu bors cause »: l'expression se rencontre à la lettre chez Platon, dans un unique passage des dialogues (Rép. X, 617e) où il est question du choix de vie qu'ont à faire les hommes avant leur réincarnation. Mais l'adjectif anaitios se retrouve, à trois reprises encore, appliquée à la divinité pour signifier que celle-ci n'est pas responsable des maux (Rép. II, 279b et c), ni de la malice dont se rendent coupables certains êtres (Tim., 42d, lois de la destinée des âmes édictées par le démiurge). Bien avant le disciple de Socrate, on rencontre, semble-t-il, l'expression daimon anaition dans un fragment mutilé d'Alcée (fr. 119 Voigt). Mais c'est dès Homère qu'apparaissent déjà les premiers germes d'une réflexion que la philosophie grecque se chargera de thématiser et que Leibniz, bien longtemps après, nommera "théodicée », entendant par là la justification d'une bonté divine que ne peut remettre en cause l'existence du mal dans le monde. Il est utile de préciser que le mot «teodicea» conserve en italien ce sens originel et particulier, alors que le mot français, tombé quelque peu en désuétude aujourd'hui, a vu sa signification s'élargir pour désigner la théologie qu'on appelle naturelle ou encore rationnelle, par opposition à la théologie dogmatique qui raisonne à l'intérieur d'une foi religieuse. La théodicée ne concerne donc qu'une partie des questions qui intéressent la théologie. Il reste que le problème de l'origine du mal et de la bonté divine, dans ses rapports avec les questions que 
posent la souffrance de l'innocent, la faute et la responsabilité humaine, la rétribution divine aussi, est au cœur des interrogations qui ont agité les hommes depuis qu'ils se sont mis à réfléchir sur le divin. Et on peut dire de ce problème qu'il restera la crux, pour ne pas dire le talon d'Achille, de toute réflexion théologique, son acuité étant d'autant plus vive que sont davantage affirmées l'absolue bonté d'un être divin créateur et sa providence.

L'A. explore donc cette thématique, riche et complexe, durant les quelques douze siècles qui séparent Homère d'Augustin, et bien au-delà encore puisque, en aval, le chapitre consacré aux néoplatoniciens fait une place à Proclus et qu'en amont, un chapitre introductif évoque, en une vingtaine de pages, les plaintes que fait entendre le Livre de Job ainsi que des traditions plus anciennes de l'Orient et de l'Égypte. Le corps principal de l'ouvrage est divisé en treize chapitres. À défaut de pouvoir en rendre compte ici en détail, j'en esquisserai simplement à gros traits le parcours. S'agissant tout d'abord des poètes, l'épopée homérique, Hésiode, la poésie lyrique et la tragédie (où apparaît notamment le thème de la souffrance nécessaire : patbei matbos) fournissent les premiers rudiments d'une théodicée. L'apport des premiers philosophes demeure, lui aussi, fort élémentaire; émergent ici les noms d'Anaximandre, avec sa notion de justice cosmique, et celui d'Anaxagore qui, par sa théorie du nồs, aurait ouvert la voie, estime l'A., à la conception d'une providence divine (pronoia), dont Socrate fut l'un des premiers représentants; Diogène d'Apollonie a droit également à une mention particulière. Un chapitre très substantiel est réservé à Platon, chez qui on peut vraiment parler d'une théodicée, même si celle-ci n'est pas coulée en forme de traité systématique; assez dispersée, la matière n'en couvre pas moins les principales ramifications du thème : bonté divine, origine du mal, responsabilité humaine, rétribution finale. Aristote, en revanche, est expédié en deux pages et demie, trop hâtivement peut-être, car ne sont pris en compte que les textes relevant de sa philosophie théorique. Or d'autres traités, particulièrement les Étbiques, fournissent de nombreuses notations théologiques qui invitent à réviser l'affirmation traditionnelle selon laquelle le Stagirite exclut toute relation allant de la divinité vers l'homme; c'est du moins la thèse novatrice que défend l'ouvrage de R. Bodéüs, Aristote et la théologie des vivants immortels, 1992, que l'A., apparemment, ne connaît pas. Les thèmes de la théodicée sont à nouveau au centre de la réflexion des Stoïciens, lesquels font coïncider la providence divine et le destin, dans une conception moniste et panthéiste que combattra le platoni cien Plutarque; ce dernier défend des perspectives nouvelles au sujet de l'origine du mal, en même temps qu'il réaffirme la bonté divine. Il n'est, on s'en doute, que très secondairement question des Épicuriens, dès lors qu'ils niaient toute providence divine. Le courant gnostique, avec sa conception d'une dégradation du monde divin, fait l'objet d'un chapitre qui précède l'exposé consacré aux néoplatoniciens, essentiellement Plotin, et aussi Proclus, comme on l'a déjà dit. Pour finir, la parole est donc donnée à un représentant de la pensée chrétienne, saint Augustin; sa conception du mal comme privation du bien ainsi que sa doctrine du péché originel ont fortement contribué à renouveler la problématique antique de la théodicée, et l'on sait de quel poids ce penseur a pesé sur les générations qui l'ont suivi. Dans ses conclusions, l'A. s'attache notamment à montrer certains prolongements qu'a connu le thème dans la philosophie moderne, de Leibnitz à Hans Jonas. L'ouvrage comporte encore une abondante bibliographie, un index des noms d'auteurs anciens et modernes ainsi qu'un index locorum.

Si cette histoire ne révolutionne pas notre connaissance de la théodicée antique, elle réussit du moins à la mettre en perspective, elle l'enrichit d'apports nouveaux et, surtout, elle l'établit sur des bases solides, en s'appuyant constamment sur les textes repérés avec soin. Diplômée en philosophie morale, mais aussi en 
philologie classique, l'A. use donc d'une méthode rigoureuse, sait analyser les textes et ordonner ses exposés avec clarté autour des thématiques propres à chaque auteur. Il est certes des points particuliers que l'on aimerait discuter, mais le principal reproche que l'on pourrait faire à l'ouvrage, me semble-t-il, c'est de manquer d'une solide introduction qui eût permis à l'A. de nouer sa problématique en précisant aussi sa terminologie ( $c f$. mes remarques initiales), d'exposer également sa démarche et de justifier certains choix effectués durant son long parcours. S'agissant de ce dernier point, je prendrai deux exemples. Chez les présocratiques, on veut bien admettre que l'unique fragment conservé d'Anaximandre ait droit à une attention particulière, compte tenu de ses implications théologiques possibles, mais on s'étonne que n'apparaisse nulle part le nom de Xénophane, en qui pourtant de nombreux fragments font voir un véritable théologien et qui a grandement pesé, à tout le moins indirectement, sur le développement d'une théodicée en dénonçant les impasses d'un anthropomorphisme qui attribue aux dieux les pires comportements des hommes. Nul, d'autre part, ne déplorera qu'un chapitre final soit réservé à un penseur chrétien, écrivant en latin, de la taille d'un saint Augustin, mais, que je sache, il n'est pas le premier penseur chrétien, tant en Orient qu'en Occident, à avoir réfléchi sur le sujet. Le fil historique est donc ici partiellement coupé; il eût été convenant à tout le moins d'en avertir le lecteur et de s'en expliquer.

Ces critiques mineures n'enlèvent rien à l'intérêt que, d'un bout à l'autre, on prend à la lecture de cette étude que recommande aussi une présentation soignée.

\section{A. Motte}

(Université de Liège)

Alain Lernould, Physique et théologie. Lecture du Timée de Platon par Proclus, Lille, Presses Universitaires du Septentrion, 2001. 1 vol. $16 \times 24 \mathrm{~cm}$, 405 p. (Problématiques pbilosopbiques). ISBN : 2-85939-644-6.

Version remaniée d'une thèse soutenue à Lille en 1992, cet ouvrage relance la question du pythagorisme du Timée de Platon, question qui fut l'objet de controverses dès l'Antiquité. Ainsi, pour le philosophe néoplatonicien Proclus, l'inspiration pythagoricienne du Timée ne fait aucun doute. L'A. entreprend précisément de montrer comment la volonté de Proclus d'attribuer un caractère pythagoricien à cette cuvre de Platon amène le Diadoque à procéder dans son Commentaire à un profond réaménagement du dialogue commenté. L'ouvrage comporte deux grandes parties. La première partie, elle-même divisée en quatre sections suivies de conclusions, étudie le but (scopos) du Timée tel qu'il est assigné par Proclus au dialogue platonicien, procède à une analyse du Commentaire dans son ensemble, met en lumière le découpage du dialogue par Proclus et les raisons qui le sous-tendent. Afin de comprendre l'arrière-plan du Commentaire, l'A. rappelle prélablement la hiérarchie des êtres selon Proclus et l'ordre de lecture des dialogues de Platon dans l'École d'Athènes du ve $s$. Le but du Timée selon Proclus est de s'attacher à la physique dans sa totalité, mais les différents moments de la création correspondent selon lui aux étapes de la remontée vers les causes divines du monde : la physique devient ainsi une sorte de théologie. De l'analyse d'ensemble du Commentaire, suivie de la présentation de la divisio textus établie par Proclus, il apparaît d'une part une parfaite concordance entre le programme que s'est fixé Proclus dans son introduction et le plan dégagé de l'ensemble du traité, d'autre part une inadéquation entre les divisions de la démiurgie dans l'In Timaeum et le plan dialectique en trois parties adopté par Platon. Le Timée de Platon ainsi recomposé et restructuré par le commentateur, 